A Four-Disc Version of the Tower of London for Clinical Use Jenny R. Tunstall ${ }^{1}$, John G. O’Gorman ${ }^{2}$, and David H. K. Shum²,

${ }^{1}$ School of Applied Psychology, Griffith University, Brisbane, Australia

${ }^{2}$ Behavioural Basis of Health, Griffith Health Institute, Griffith University, Brisbane, Australia

Abstract word count: 184

Manuscript word count (exc. figures/tables): 5718

* Correspondence should be addressed to David H. K. Shum, School of Applied Psychology (Mt Gravatt Campus), Griffith University, Mt Gravatt, Queensland 4122, Australia, Phone: +61 75678 7629, Fax: +61 73735 3388, Email: d.shum@griffith.edu.au.

\title{
Acknowledgements
}

The authors wish to acknowledge all of the participants involved in these studies, as well as to extend a special thank you to the children who were involved, and their parents for their participation. 


\begin{abstract}
Three studies are reported on the development of a four-disc version of the Tower of London test of planning ability. The first $(n=138)$ involved the selection of items based on rational and empirical criteria to provide a short test of graded difficulty suitable for use with children and clinical populations. The second study $(n=480)$ checked the properties of the 10 -item test on a new sample and in addition examined the internal consistency and factor structure of the test. The third study ( $n=61$ ) examined the test-retest reliability of the test over a period of one month. The difficulty level of the test remained relatively stable from sample to sample and was sensitive to linear trend in performance from age 5 years up to 30 years. Total score did not reflect the action of a single underlying construct but rather appeared to index a number of factors. Scores were reasonably stable over the one-month period studied, at least for the children’s sample employed. The four-disc version is a promising method of assessing planning in children and adolescents in clinical situations.
\end{abstract}




\section{A Four-Disc Version of the Tower of London for Clinical Use}

The Tower of London (TOL) is used in neuropsychological assessment for the diagnosis of a variety of neurological conditions (Sullivan, Riccio, \& Castello, 2009). Originally devised by Shallice (1982) to assess frontal lobe function, the TOL is now usually considered a test of the executive function of planning. In its original form it presents the test taker with three vertical pegs of different heights, three discs of different colours that can be threaded on to the pegs, and the requirement to move the discs according to a set of rules from a given starting configuration (e.g., red, green, and blue on different pegs) to an end or goal configuration (e.g., all three on the one peg, with green on top and red on the bottom).

The use of the original TOL as a neuropsychological test has had to contend with two major limitations, lack of sensitivity to changes in age and lack of reliability. Early attempts to standardize the TOL to improve its utility for neuropsychological assessment showed a ceiling effect on scores (Anderson, Anderson, \& Lajoie, 1996; Krikorian, Bartok, \& Gay, 1994), with no further improvement on the test occurring after about age 12. Such a ceiling effect reduced the sensitivity of the test to closed head injury in older children and was not consistent with the view that executive functions improve from childhood through adolescence into early adulthood (Levin et al., 1997).

Attempts to improve the sensitivity of the TOL were directed to increasing the difficulty of the task. In 2002, Berg and Byrd reviewed the variants of the TOL published to that date. These included versions that altered the time allowed to complete an item or changed the scoring procedures, as well as versions that increased the number of moves required for successful completion of an item, the number of items, the number of pegs, or the number of discs. Berg and Byrd observed that some of the changes introduced with these variants do not change the 
problem structure of the original TOL, but those that increase the number of pegs or the number of discs do.

Kafer and Hunter (1997) increased both the number of discs and the number of pegs to four. Planning time was found to be shorter and the number of moves required fewer with this version compared to the original, which does not recommend it as a way of increasing sensitivity. As Berg and Byrd (2002) pointed out, the problem space of the original TOL has three discs and three empty spaces on the pegs, which is the minimum possible for the problem to be solved. The Kafer and Hunter version creates a problem space with four discs and six empty spaces, a less difficult problem space. Ward and Allport (1997) used four-disc and fivedisc versions with adult samples in experiments directed to understanding the underlying planning process involved. Importantly, they used pegs of equal height that, in the case of the five disc version, creates a problem space with a ratio of 5 discs to 15 spaces, which is quite different to the ratio of $1: 1$ used in the original TOL.

The second psychometric problem involves internal consistency and stability of scores on the TOL. Humes, Welsh, Retzlaff, and Cookson (1997) reported a Cronbach alpha of .25 for the original version with a sample of 61 adults. Lowe and Rabbitt (1998) tested a large sample of 60 to 80 year olds on the CANTAB, a computer-based suite of neuropsychological tests that includes a version of the original TOL. They reported test-retest coefficients over a four-week period ranging from .26 to .68, depending on the score used. Lemay, Bédard, Rouleau, and Tremblay (2004) examined retest performance over two consecutive intervals of 14 days for a sample of 19 middle aged to elderly participants. Intra-class correlations over the three intervals varied with the index used from 0 to .83. Kaller, Unterrainer, and Stahl (2012) studied 13018 to 32 year olds using a version of the original TOL that was based on a careful analysis of the 
parameters of the task, such as the number and nature of optimal and sub-optimal solution paths. They reported an average split-half reliability of .72 and an alpha of .69 for a 32-item test.

As these more recent studies show, the internal consistency problem can be solved by increasing the number of items in the task. A longer form of the original version can also show the expected age change in performance. Albert and Steinberg (2011) used a 20-item computerbased version of the original TOL with a sample ranging in age from 10 to 30 years $(n=890)$. They showed statistically significant linear and quadratic trends in TOL score with increasing age, reflecting an upward trend in performance with age that leveled out in the adult years. Unterrainer et al. (2014) used a 24-item version of the original TOL with a sample of 178 4-13 year olds and found a linear increase in performance with age.

The aim of the present study was to develop a shorter form of TOL but one with reasonable reliability. A shorter form would allow test takers more than one opportunity for problem solution without overly extending testing time. This would provide an efficient and informative clinical assessment of planning.

Our approach was to increase the number of discs to four but remain with three pegs and to adjust the height of the pegs to accommodate one additional disc. This created a problem space with four discs and five empty spaces, close to the 1:1 ratio of the Shallice (1982) TOL, but with a possible 264 goal configurations from a given starting configuration. It must be acknowledged, however, that the number of empty spaces is only a rough guide to difficulty. More empty spaces increase the potential for multiple solutions and reduce alternative dead-end solutions, but it is structural parameters such as goal hierarchy and search depth that are of more direct relevance (Kaller, Rahm, Kostering, \& Unterrainer, 2011). As with the original TOL, the task is not computer-based but we took the opportunity of changing the colours of the discs from 
red, green, and blue to black, white, yellow, and mid-blue. Approximately $10 \%$ of the male population has difficulties with colour vision (Huffman, Vernoy \& Vernoy, 1997) but the colours we chose do not pose a discrimination problem for these individuals.

Working with this four-disc version, we used standard psychometric criteria (difficulty level, item-total correlation) to select items from the larger pool of possible items to create a version which was reasonably short (10 items) but with adequate reliability. Data for three samples are reported. Study 1 involved initial construction with child and adult participants. Study 2 was directed to confirming the results of Study 1 with a larger sample and examining the questions of internal consistency and factor structure. Study 3 was a smaller scale study that took up the question of retest stability of scores. We hypothesized that performance on a reliable measure of planning would show a linear trend with age, consistent with previous findings referred to above, and would be differentiable from scores on an intelligence test, given that executive functions such as planning are not wholly explained by IQ (e.g., Arffa, 2007).

\section{Study 1}

We sought a final item set of 10 to 12 items that could be arranged in order of difficulty from problems that could be solved by young children to those that would be appropriate in late adolescence. Three criteria were applied in selecting problems. The first was to have items that varied in the number of moves required so as to provide a range of difficulty. Although items with more moves are not invariably more difficult than items with fewer moves, number of moves was considered a reasonable guide to ensure a range of difficulty. Four items at each move level from 2 moves to 9 moves were selected and one 10-move item added. Second, items with few solution paths were chosen to minimise instances of 'saving' a failed plan by moving to a solution along a different path. The third criterion was that there was to be a mixture of tower- 
ending and flat-ending items. Tower-ending items (discs stacked on top of each other) have been shown (Klahr, 1978) to be generally easier than flat-ending items (discs spread across the pegs). An initial set of 33 items were selected in this way and subject to empirical analysis.

\section{Participants}

The sample comprised 65 children (28 males), aged between 4 and 9 years $(M=6.92$ years, $S D=1.08)$, and 73 adults (30 males), aged between 17 and 82 years $(M=43.62, S D=$ 19.96). Children were recruited from schools and after-school care facilities and adults were recruited from an undergraduate course in psychology, from senior citizens associations, and from the general community, all in the Brisbane, Australia area. Those who reported a neurological condition or were taking medication that might interfere with cognitive performance were excluded. All adult participants gave informed consent and in the case of children their parents gave informed consent. Recruiting and testing were in accord with the ethical standards in place in the University at the time.

\section{Materials}

The tower consisted of a wooden base (225 x 57 x $18 \mathrm{~mm}$ in dimensions) with four pegs $15 \mathrm{~mm}$ in diameter spaced $55 \mathrm{~mm}$ apart. Heights of the four pegs were 80, 62, 42, and $26 \mathrm{~mm}$. A cover was placed over the shortest peg when used as the four-disc version reported on here. There were four wooden discs, $50 \mathrm{~mm}$ in diameter and $20 \mathrm{~mm}$ high, in which there was cut a central annulus that allowed easy movement on and off a peg. Colours of the four discs were black, white, yellow, and mid-blue. The goal state for each problem was specified in a coloured diagram, 68 x 34 mm (see Supplementary Figure 1 for a graphical representation of the start and goal positions for the final 10 items selected for the TOL4). Only one diagram was visible for an item. 


\section{Procedure}

The pool of 33 items was divided into two sets, each with 17 items (two for each of the 2 to 9 move levels plus the 10 move item) and within each set ordered in terms of the number of moves required for solution. Within each move level the order of the two items was counterbalanced to control for order effects, which resulted in four sets of 17 items.

Instructions for administration differed for adults and children. There were no instructions as to when to commence and unlimited time was allowed for each item. Instructions emphasized accuracy over speed. Rule breaks were penalized after the first instruction. Three trials were allowed for each item. The scoring procedure adopted was based on that used by Krikorian et al. (1994), with three points awarded for correctly solving the item on the first attempt, two points for solution on the second attempt, one point for solution on the third attempt, and zero points for failure to solve the item within three attempts.

All data analyses in this and subsequent studies used SPSS Version 22, unless explicitly stated otherwise.

\section{Results and Discussion}

First, the possibility of gender differences in performance was checked by comparing total scores for males and females. The mean for males $(M=28.36, S D=8.68)$ was found not to differ from that for females $(M=29.60, S D=8.63), t(1,136)=0.83, p=.41$. For the adult sample, the mean for males $(M=35.37, S D=5.27)$ was not significantly different from that for females $(M=36.47, S D=5.06), t(1,71)=0.90, p=.37$. For the children's sample, the mean for males $(M=20.86, S D=4.05)$ was not significantly different from that for females $(M=21.43$, $S D=2.59), t(1,63)=0.70, p=.49$. Accordingly, all subsequent analyses combined the two genders. 
There was a ceiling effect for the adults’ scores for items requiring fewer than 6 moves and for this reason only the children's data were used in selecting the 2, 3, 4, and 5 move items. The children, on the other hand, evidenced a floor effect for problems that required 6 moves or more and hence only the adults' data were used in selecting these items. Four-year olds not attending school had the most difficulty, even with 2-move items, as evidenced by frequent rulebreaking.

Item analysis involved consideration of mean scores on the items, which were used as an index of difficulty, and association of item score with total score across all items, which was used as an index of the ability of the item to discriminate high and low scorers. Both comparisons of item scores for those in the top and bottom quartile of total score and product moment correlation of item and total scores were used. Items were sought that provided a range of difficulty and showed good discrimination. A final selection criterion imposed was that when arranged in increasing order of difficulty the items increased in terms of the number of moves required. An orderly progression of test difficulty in terms of the number of moves required was thought to be less disconcerting than having number of moves vary up and down as difficulty increased. It was also considered important to provide an opportunity in practical assessment situations for the tester to discontinue if a participant could not complete two consecutive items in at least three trials.

Descriptive statistics for each of the items finally chosen are presented in Table 1 (see Supplementary Table 1 for the item-difficulties and item-correlations for all items). The itemtotal correlations are for each item with the total scores across all 33 items. Also included is the correlation of the item score with participant's age. 


\section{[INSERT TABLE 1 HERE]}

As inspection of Table 1 indicates, the attempt to provide a test ordered in terms of item difficulty was largely successful. Item 7, however, proved to have a lower difficulty level than expected as indicated by its higher mean item score and relatively smaller variance. These item statistics point to the item being better located between Items 3 and 4 . Its position was maintained, however, in the interests of having items ordered in terms of the number of moves required. (An alternative pointed out by a reviewer of an earlier version of the paper is to exchange the order of Items 6 and 7.)

All items correlated substantially with total score on the test and in all cases the coefficients were significantly different from zero. This of course is a minimal requirement for considering all the items to be measuring the same construct. Only two of the items showed a statistically significant correlation with age and these were for the children's sample. For the children the correlations were, however, more substantial than for adults, which may reflect a sharper growth function in planning ability over childhood compared with adulthood.

\section{Study 2}

The purpose of the second study was to check the findings of the first with respect to the performance of the selected items using an independent and larger sample and to examine the internal structure of the test in terms of internal consistency and factor structure. It was expected that absolute item difficulties would remain approximately the same as for the first study and that relative item difficulties would remain constant. Further, it was expected that internal consistency would be at least .70 and ideally greater than .90, the lower limits proposed by 
Nunnally (1994). Because there was no previous research that examined the factor structure of the test we employed exploratory factor analysis.

\section{Participants}

Recruiting procedures were the same as described in Study 1. In this study, there were a total of 146 adults (64 males) aged 17 to 53 years $(M=27.73, S D=9.83)$ and 334 children $(187$ males) aged 5 to 15 years $(M=9.96, S D=3.04)$.

\section{Materials}

The four-disc version of the Tower of London as described in Study 1 was used, with the 10 items selected on the basis of the item analysis reported in that study. A measure of verbal intelligence, the Vocabulary subtest of the Kaufman Brief Intelligence Scale (Kaufman \& Kaufman, 1990), was included. This could only be obtained for 67 participants in the adult sample. The scale score on the subtest was used for analysis.

\section{Procedure}

Instructions, administration, and scoring were as described in the first study. There were, however, three additional measures included in the analysis. The first, termed planning time, was the average time, over all items attempted, from commencement of the trial to the first release of a disc on that trial. Planning time was scored for the first trial only for each item. The second measure was the total number of items solved in the minimum number of moves on the first trial for each item. The possible range was 0 (none solved on the first attempt) to 10 (all problems solved on the first attempt). The third was the total number of items solved in the minimum number of moves, within three attempts. The possible range here was 0 (no problems solved) to 10 (all problems solved). 


\section{Results and Discussion}

There were no differences in total score between genders. For the adult sample the mean for males was $22.63(S D=2.50)$ and for females was $22.46(S D=2.80), t(144)=0.40, p=.692$. For the children, the mean for males was $19.01(S D=3.69)$ and for females was $18.27(S D=$ 3.93), $t(332)=1.78, p=.076$.

Tables 2 and 3 summarise the results of item analyses conducted separately for the child and adult samples. Inspection of these tables indicates that the difficulty level of the items matches their ordinal position for the children's sample. Item 7 that appeared in Study 1 to be an easier item than its ordinal position indicated appears in Table 2 to be correctly placed. For the adult sample, inspection of Table 3 suggests that this item might be better placed between Items 5 and 6 . Tables 3 and 4 confirm the observation in the previous study that items correlate with total score, with the exception of Item 1 in the children's sample and Items 1 and 2 in the adult sample. The low correlations for these items are a function of their low variance, with most of the children being able to complete Item 1 and most adults Items 1 and 2. Inclusion of an easy item to begin a test does have the benefit of encouraging the test-taker at the outset.

[INSERT TABLE 2 HERE]

[INSERT TABLE 3 HERE]

Tables 2 and 3 also confirm the observation in the first study that item scores are correlated with age for the children's sample but not for the adults. This is consistent with a rapidly advancing capacity for planning during the childhood years that reaches a peak in early 
adulthood (see below). Tables 2 and 3 provide information on correlations with verbal intelligence and indicate that for most items in both samples these are not significantly different from zero. This implies that the capacities captured by the test are not redundant with those commonly measured by verbal IQ tests.

Item means for Study 2 were compared with those for Study 1, using Welch's test because of the differences in sample sizes and variances. With 10 comparisons and using the Bonferroni correction, an alpha level was set at .005. For Items 4, 5, 6 and 8, difficulty level was lower in Study 2 than Study 1 and the differences were statistically significant. However, with one exception (Item 7), the rank order of item difficulties was the same between the two studies.

To examine the relation of total score to age, a regression analysis was performed using those aged 30 or younger in the adult and child samples. The age of 30 was used because any development of executive function would be expected to have peaked by then. Both linear and quadratic components were statistically significant, $F_{\text {linear }}(1,428)=144.40, p<.001, R^{2}=.25$; $F_{\text {quadratic }}(2,427)=97.16, p<.001, R^{2}=.31$. The regression equation was TOL 4 Total Score $=$ $10.63+1.07 x$ Age -.03xAgeSquared. The linear component was clearly the stronger. Table 4 presents the means and standard deviations for the combined sample of children and adults split into age groups. The results of the regression analysis are similar to those reported by Albert and Steinberg (2011) with a longer form of the TOL, with performance improving with age but leveling out in the adult years.

[INSERT TABLE 4 HERE] 
Table 5 presents the descriptive statistics and intercorrelations for the additional measures of performance on the TOL as well as for total score, and the correlations of all measures with age and verbal IQ. Inspection of the table indicates that, of the additional measures, the minimum number of moves on the first trial for each item and the total number of items solved in the minimum number of moves correlate with each other and with total score in both samples. The relationship is, however, less than perfect, which points to the value of using three trials per item. Using only one trial per item may lead to a conclusion at variance with that based on three trials. It is the planning time measure, however, that appears most different from the other three and may provide additional information to that contained in total score. As for the correlations with age and verbal intelligence, all TOL measures correlated with age in the children's sample but none correlated with age in the adult sample. In neither sample did any of the TOL measures correlate with verbal intelligence.

\section{[INSERT TABLE 5 HERE]}

In assessing internal consistency of the 10-item test, Cronbach’s coefficient alpha was computed separately for the two samples. To examine the effect of the 3-point scoring system, alpha was computed both for the test when scored based on all three trials and when based on dichotomous scoring of performance on the first trial only for each item (correct/incorrect). Given the concerns expressed in the literature about coefficient alpha (see e.g., Dunn, Baguley, \& Brunsden, 2013; Sijtsma, 2009), three other indices of reliability were calculated: the greatest lower bound (glb; Sijtsma, 2009), omega (Revelle \& Zinbarg, 2009), and lambda 4 (Guttman, 1945). These were calculated using the psych package developed for the $\mathrm{R}$ environment by 
Revelle (2014; http://cran.r-project.org/web/packages/psych/psych.pdf). All coefficients were calculated for the 3-point and the dichotomous scoring systems. Results are summarized in Table 6.

\section{[INSERT TABLE 6 HERE]}

Inspection of Table 6 indicates that estimates are higher for the children's sample than for the adult sample, are higher for the 3-point than for dichotomous scoring, and higher for the alternative estimates than for alpha. The higher estimates of reliability for the children's sample probably reflect the greater variability in performance for this sample than for the adult sample, which in turn is likely a function of the difficulty level of the items for the two age groups. This would suggest use of the test with younger age levels. The higher reliabilities for the 3-point scoring system again is likely a result of the greater variability in scoring, which points to a greater sensitivity of the test when scored in this way. The differences with the different indices of reliability indicate that the test, as with all psychological tests, cannot be said to have one reliability. The estimate depends on the sample employed and on the extent to which assumptions of particular indices are met. It has been argued (e.g., Dunn et al., 2013) that the assumptions of the alpha coefficient are quite restrictive and often not met in practice, whereas those for the alternative indices employed here are more realistic. For the 3-point system of scoring, these provide estimates that are quite close for both the children's and the adult samples, with the lambda 4 estimate lying between the other two. Lambda 4 can be thought of as the optimal split-half reliability and in terms of true score theory indicates that approximately $65 \%$ of the variance in scores for the children's sample and approximately $50 \%$ of variance in scores for the adult sample as being due to true score variance. 
A careful examination of the effects of deleting items indicated very little change in indices of reliability. For example, only in the case of Item 1 in the children's sample and Items 1 and 9 in the adult sample would the deletion of the item increase the alpha by more than the third decimal place. Item 1 acts as a practice item and its retention on these grounds was considered warranted.

Previous studies have shown that internal consistency of versions of the TOL is suspect unless a large number of items (e.g., 30) are included (cf, Humes et al., 1997; Kaller et al., 2012). Results with the 10-item version indicate reasonable reliability, for the children's sample at least. Internal consistency is, of course, not the only or necessarily best way to establish reliability in all cases, as discussed below.

Exploratory factor analysis was undertaken for each sample, using a principal factors solution with rotation by oblimin. For the children's sample the average inter-item correlation was .11 and the Kaiser-Meyer-Olkin (KMO) index of sampling adequacy was .69, indicating that it was reasonable to factor analyse. The analysis showed that three factors had eigenvalues greater than 1 . The scree plot was consistent with a three-factor solution, which accounted for $45 \%$ of the variance. The first factor loaded Items 5 to 10 at better than .30 and was defined by Item 8 (.75) and to a lesser extent Item 10 (.63). The second factor loaded Items 1, 5, and 6 and the third factor loaded Items 2, 3, 4, and 6. The clearest separation is between the first and third factors, which may reflect different components of planning, with the more difficult items possibly requiring a greater involvement of working memory.

For the adult sample the average inter-item correlation was .04 and the KMO was only .48, which questioned the appropriateness of attempts to factor. An attempt was made, however, 
to proceed with an analysis. Five factors had eigenvalues greater than 1 but a five-factor solution was not confirmed by inspection of the scree plot and an attempt to rotate five factors failed.

The results of the factor analyses indicate that the 10-item test for both the adult and children's samples is multidimensional and that the single total score cannot be considered to reflect expression of a single underlying trait. This is not really surprising because planning involves a complex cognitive process which is unlikely to be reducible to a single factor. Therefore, total score is best thought of as an index that simply aggregates examples of planning behavior rather than as a scale. If this is a reasonable analysis, then the appropriate index of reliability is not internal consistency but rather test-retest stability of total score. The final study examined this property of TOL-4.

\section{Study 3}

In this study, the TOL-4 as previously described was administered to two further samples, one a sample of adults and the other of children, on two occasions spaced at an interval of 1 month. Change in mean score over time and the correlation between total scores on the two occasions were examined.

\section{Participants}

Recruiting procedures were the same as described in Study 1. In this study, there were 40 adults (21 males) aged 21 to 55 years $(M=34.47, S D=9.22)$ and 21 children (9 males) aged 5 to 15 years $(M=10.10, S D=3.33)$.

\section{Materials}

The four-disc version of the Tower of London as described in the previous study was used for both administrations. 


\section{Procedure}

Instructions, administration, and scoring were as described in the first study. Testing conditions were the same for both administrations that were spaced as close to one month apart as practicable $(M=32.50$ days, $S D=3.84)$.

\section{Results and Discussion}

For the children's sample the mean and SD for the first session were 17.05 (4.80) and for the second session they were $19.14(3.72), t(2)=3.36, p=.003$. The Pearson correlation of scores on the two occasions was .80, 95\% CI [.52, .92]. For the adult sample the mean and SD for the first occasion of testing were $22.45(S D=2.62)$ and for the second occasion were 22.73 (1.94), $t(39)=.719, p=.476$. The Pearson correlation of scores across occasions was .47, 95\% CI $[.14, .71]$

A practice effect was apparent for children, with performance on the second occasion being better than that on the first to a statistically significant degree. No such practice effect was apparent in the data for the adult sample, although, it should be noted, the variance of scores on the second occasion was almost half that of the first. There was a statistically significant correlation for total score across occasions for both samples, but only for the child sample was it of a magnitude that would be considered acceptable (e.g., Nunnally, 1994). The lower correlation for the adult sample was probably due to the reduced variance of scores on the second occasion. A comparison of scatter plots of scores on the two occasions separately for the children's and the adult samples supported this conclusion (see Supplementary Figure 2).

The Pearson correlation has been criticised as an index of test-retest reliability because it does not take into account systematic error, which for the children’s sample was present, given 
the statistically significant increase in test score from the first to the second occasion. For this reason, intraclass correlations (ICCs) were also calculated. Of the possible ICCs described by Shrout and Fleiss (1979), ICC $(2,1)$ and $\operatorname{ICC}(3,1)$ were selected. The former includes estimation of random and fixed sources of variance in the error term (between occasions and between participants) and can be considered to index absolute agreement of test scores, whereas the latter includes only random sources (between participants) and can be considered an index of consistency.

For the children's sample, ICC $(2,1)$ was .70, 95\% CI $[.41, .90]$ and for the adult sample was .45, 95\% CI [.17, .68]. For the children's sample, ICC $(3,1)$ was .78, 95\% CI $[.53$, .91] and for the adult sample was .45, 95\% CI [.16, .67]. That the two ICCs do not differ in the case of the adult sample is a reflection of the fact that there was no systematic effect of occasions with this sample. The smaller ICC for the adult sample compared with the children's sample is most likely a result of the difference in variance for the two samples. As inspection of the SDs for the two samples indicates, the variance for the children's sample was more than three times that for the adult sample on each occasion.

\section{General Discussion}

The present study was undertaken to provide a reliable version of the TOL that was sensitive to the increase in executive functioning throughout the child and adolescent years. The 10-item TOL-4 reported here provides a problem space broadly similar to the original TOL-3 with a stable, graded order of difficulty from Item 1 to Item 10. Total score on the TOL-4 increases from age 5 up to late adolescence and early adulthood in a largely linear fashion, and thus shows its sensitivity to growth in executive function over the adolescent years as well as those of childhood. Underlying factor structure of the test is complex and total score is therefore 
best thought of as an aggregate index rather than a measure of a single construct. Test-retest reliability of the total score on the test when used with a sample of children and adolescents is adequate, although less than the lower bound of .9 usually required for individual testing. The test has been designed for use with children and appears too easy for use with normally functioning adults. The lower level of difficulty with adults reflected in reduced variance probably accounts for the poor evidence of reliability with these samples and underlines the need to restrict application of the test to samples for which it was designed. The test is likely to have value, however, with clinical samples where performance inferior to normal adult functioning might be expected. The use with these samples of the 3-point scoring system based on three trials per item is likely to provide a more searching assessment of the limits of planning ability. Provision of more than one trial provides an opportunity to learn from error and benefit from feedback, and provides higher estimates of reliability than is the case with a single-trial, dichotomous scoring system. As the analysis in Study 2 showed, the number of items completed correctly on the first trial is an imperfect indicator of the number completed correctly after three trials.

The clinical utility of the TOL-4 has been demonstrated in studies with patients with traumatic brain injury (TBI; Shum, Tunstall, O’Gorman, \& Maujean, 2003; Shum et al., 2009). For example, Shum et al. (2009) showed that TBI patients had lower total scores on the TOL-4 than control participants matched on age, gender, and years of education. The effect size for the difference between patients and controls was in Cohen's terms 'medium' to 'large' $(d=.62)$. Patients with localized frontal lesions as established by CT scan $(n=15)$ differed from those without such lesions $(n=16)$ in terms of total score, with a 'large' effect size $(d=.82)$. 
Importantly, the length of the test was such that patients maintained engagement for the duration of the testing session.

Further work with the test will need to establish where it is located with respect to other cognitive functions. The present data point to its relative independence from verbal ability but do not address its relationship with other executive functions. Planning is unlikely to be completely independent of other functions, importantly working memory. As noted above, some of the items towards the end of the test may place a heavier demand on working memory than those early in the test. However, too large an overlap of variance on the TOL-4 with measures of other executive functions would compromise a claim for it as an index of planning alone. 


\section{References}

Albert, D., \& Steinberg, L. (2011). Age differences in strategic planning as indexed by the Tower of London. Child Development, 82(5), 1501-1517. doi:10.1111/j.1467-8624. 2011.01613.x

Anderson, P., Anderson, V., \& Lajoie, G. (1996). The Tower of London Test: Validation and standardization for pediatric populations. The Clinical Neuropsychologist, 10(1), 54-65. doi:10.1080/13854049608406663

Arffa, S. (2007). The relationship of intelligence to executive function and non-executive function measures in a sample of average, above average, and gifted youth. Archives of Clinical Neuropsychology, 22(8), 969-978. doi:10.1016/j.acn.2007.08.001

Berg, K. W., \& Byrd, D. L. (2002). The Tower of London spatial problem-solving task: Enhancing clinical and research implementation. Journal of Clinical and Experimental Neuropsychology, 24(5), 586-604. doi:10.1076/jcen.24.5.586.1006

Dunn, T. J., Baguley, T., \& Brunsden, V. (2013). From alpha to omega: A practical solution to the pervasive problem of internal consistency estimation. British Journal of Psychology, 105(3), 399-412. doi:10.1111/bjop.12046

Guttman, L. (1945). A basis for analyzing test-retest reliability. Psychometrika, 10(4), 255-282. doi: 10.1007/BF02288892

Huffman, K., Vernoy, M., \& Vernoy, J. (1997). Psychology in action (4th ed.). Boston, MA: Duxbury.

Humes, G. E., Welsh, M. C., Retzlaff, P., \& Cookson, N. (1997). Towers of London and Hanoi: Reliability of two executive function tasks. Assessment, 4(3), 249-257. 
Kafer, K. L., \& Hunter, M. (1997). On testing the face validity of planning/problem solving tasks in a normal population. Journal of the International Neuropsychological Society, 3(2), 108-119.

Kaller, C. P., Rahm, B., Kostering, L., \& Unterrainer, J. M. (2011). Reviewing the impact of problem structure on planning: A software tool for analyzing tower tasks. Behavioural Brain Research, 216(1), 1-8. doi:10.1016/j.bbr.2010.07.029

Kaller, C. P., Unterrainer, J. M., \& Stahl, C. (2012). Assessing planning ability with the Tower of London Task: Psychometric properties of a structurally balanced problem set. Psychological Assessment, 24(1), 46-53. doi:10.1037/a0025174

Kaufman, A. S., \& Kaufman, N. L. (1990). Kaufman Brief Intelligence Test. Circle Pines, MN: American Guidance Service.

Klahr, D. (1978). Goal formulation, planning, and learning by pre-school problems solvers or: “My socks are in the dryer”. In R. S. Siegler (Ed.), Children’s thinking: What develops? (pp. 181-212). Hillsdale, NJ: Erlbaum.

Krikorian, R., Bartok, J., \& Gay, N. (1994). Tower of London procedure: A standard method and developmental data. Journal of Clinical and Experimental Neuropsychology, 16(6), 840850. doi:10.1080/01688639408402697

Lemay, S., Bédard, M-A, Rouleau, I., Tremblay, P-L. (2004). Practice effect and test-retest reliability of attentional and executive tests in middle-aged to elderly subjects. The Clinical Neuropsychologist, 18(2), 284-302. doi:10.1080/13854040490501718

Levin, H. S., Song, J., Scheibel, R. S., Fletcher, J. M., Harward, H., Lilly, M., \& Goldstein, F. (1997). Concept formation and problem-solving following closed head injury in children. Journal of the International Neuropsychological Society, 3(6), 598-607. 
Lowe, C., \& Rabbitt, P. (1998). Test/re-test reliability of the CANTAB and ISPOCD neuropsychological batteries: Theoretical and practical issues. Neuropsychologia, 36(9), 915-923. doi:10.1016/S00283932(98)00036-0

Nunnally, J. C. (1994). Psychometric theory (3rd ed). New York, NY: McGraw Hill.

Revelle, W., \& Zinbarg, R. E. (2009). Coefficients alpha, beta, omega, and the glb: Comments on Sijtsma. Psychometrika, 74(1), 145-154. doi:10.1007/s11336-008-9102-z

Shallice, T. (1982). Specific impairments of planning. Philosophical Transactions of the Royal Society of London, B298(1089), 199-209. doi:10.1098/rstb.1982.0082

Shrout, P. E., \& Fleiss, J. L. (1979). Intraclass correlations: Uses in assessing rater reliability. Psychological Bulletin, 86(2), 420-428. doi:10.1037/0033-2909.86.2.420

Shum, D., Gill, H., Banks, M., Maujean, A., Griffin, J., \& Ward, H. (2009). Planning ability following moderate to severe traumatic brain injury: Performance on a 4-disc version of the Tower of London. Brain Impairment, 10(3), 320-324. doi:10.1375/brim.10.3.320

Shum, D., Tunstall, J., O’Gorman, J. G., \& Maujean, A. (2003). Effects of traumatic brain injury on planning ability. Journal of the International Neuropsychological Society, 9, 234.

Sijtsma, K. (2009). On the use, the misuse, and the very limited usefulness of Cronbach's alpha. Psychometrika, 74(1), 107-120. doi:10.1007/s11336-008-9101-0

Sullivan, J. R., Riccio, C. A., \& Castello, C. L. (2009). Concurrent validity of the Tower tasks as measures of executive function in adults: A meta-analysis. Applied Neuropsychology, 16(1), 62-75. doi:10.1080/09084280802644243

Unterrainer, J. M., Kaller, C. P., Loosli, S. V., Heinze, K., Ruh, N., Paschke-Muller, M, Rauh, R., Biscaldi, M., \& Rahm, B. (2014). Looking ahead from age 6 to 13: A deeper insight 
into the development of planning ability. British Journal of Psychology. doi:10.1111/ bjop.12065

Ward, G., \& Allport, A. (1997). Planning and problem solving using the five disc Tower of London task. The Quarterly Journal of Experimental Psychology Section A: Human Experimental Psychology, 50(1), 49-78. doi:10.1080/713755681 


\section{Tables}

Table 1

Descriptive Statistics for the Items Chosen and Correlation of Item Scores with Age

\begin{tabular}{|c|c|c|c|c|c|}
\hline Item & Moves & Mean & $S D$ & $r_{\text {total }}$ & $r_{\text {age }}$ \\
\hline \multicolumn{6}{|c|}{ Children $(n=65)$} \\
\hline 1 & 2 & 2.91 & 0.29 & 0.55 & 0.32 \\
\hline 2 & 3 & 2.79 & 0.64 & 0.78 & 0.25 \\
\hline 3 & 4 & 2.56 & 0.93 & 0.65 & 0.44 \\
\hline 4 & 5 & 2.32 & 1.07 & 0.66 & 0.31 \\
\hline 5 & 5 & 2.03 & 1.14 & 0.62 & 0.45 \\
\hline \multicolumn{6}{|c|}{ Adults $(n=73)$} \\
\hline 6 & 6 & 2.00 & 1.20 & 0.63 & -0.10 \\
\hline 7 & 6 & 2.50 & 0.91 & 0.45 & -0.14 \\
\hline 8 & 7 & 1.56 & 0.25 & 0.58 & -0.05 \\
\hline 9 & 9 & 0.85 & 0.97 & 0.70 & -0.21 \\
\hline 10 & 9 & 0.17 & 0.51 & 0.49 & -0.24 \\
\hline
\end{tabular}

Note. Items in bold are statistically significantly different from zero at $p<.05$ two-tailed. 
Table 2

Descriptive Statistics for the Items Chosen and Correlations of Item Scores with Age and Verbal IQ for the Children's Sample $(n=334)$

\begin{tabular}{ccccccc}
\hline Item & Moves & Mean & $S D$ & $r_{\text {total }}$ & $r_{\text {age }}$ & $r_{\text {IQ }}$ \\
\hline 1 & 2 & 2.98 & 0.15 & 0.06 & -0.02 & 0.03 \\
2 & 3 & 2.90 & 0.36 & $\mathbf{0 . 3 2}$ & $\mathbf{0 . 2 3}$ & -0.04 \\
3 & 4 & 2.85 & 0.47 & $\mathbf{0 . 3 2}$ & $\mathbf{0 . 2 5}$ & 0.02 \\
5 & 5 & 2.75 & 0.68 & $\mathbf{0 . 4 4}$ & $\mathbf{0 . 2 7}$ & 0.09 \\
6 & 5 & 2.46 & 0.84 & $\mathbf{0 . 4 4}$ & $\mathbf{0 . 2 0}$ & -0.08 \\
7 & 6 & 2.08 & 1.15 & $\mathbf{0 . 5 9}$ & $\mathbf{0 . 2 6}$ & 0.05 \\
8 & 6 & 1.37 & 1.26 & $\mathbf{0 . 6 2}$ & $\mathbf{0 . 2 2}$ & 0.06 \\
9 & 7 & 0.93 & 1.21 & $\mathbf{0 . 6 2}$ & $\mathbf{0 . 2 7}$ & $\mathbf{0 . 1 1}$ \\
10 & 9 & 0.19 & 0.65 & $\mathbf{0 . 3 2}$ & 0.06 & 0.04 \\
\hline
\end{tabular}

Note. Items in bold are statistically significantly different from zero at $p<.05$, two-tailed.

${ }^{\mathrm{a}}$ For $r_{\mathrm{IQ}}, n=330$. 
Table 3

Descriptive Statistics for the Items Chosen and Correlations of Item Scores with Age and Verbal IQ for the Adult's Sample $(n=146)$

\begin{tabular}{ccccccc}
\hline Item & Moves & Mean & $S D$ & $r_{\text {total }}$ & $r_{\text {age }}$ & $r_{\text {IQ }}$ \\
\hline 1 & 2 & 2.97 & 0.18 & -0.03 & -0.09 & 0.05 \\
2 & 3 & 2.94 & 0.24 & 0.06 & 0.16 & -0.04 \\
4 & 4 & 2.92 & 0.34 & $\mathbf{0 . 1 8}$ & 0.10 & 0.08 \\
5 & 5 & 2.86 & 0.44 & $\mathbf{0 . 4 0}$ & -0.14 & 0.22 \\
6 & 5 & 2.74 & 0.55 & $\mathbf{0 . 4 5}$ & -0.08 & 0.02 \\
7 & 6 & 2.53 & 0.82 & $\mathbf{0 . 3 3}$ & 0.04 & 0.03 \\
8 & 6 & 2.58 & 0.86 & $\mathbf{0 . 4 0}$ & -0.05 & -0.05 \\
9 & 7 & 2.14 & 1.20 & $\mathbf{0 . 4 6}$ & 0.00 & 0.02 \\
10 & 9 & 0.84 & 1.18 & $\mathbf{0 . 3 0}$ & -0.01 & $\mathbf{- 0 . 2 7}$ \\
\hline
\end{tabular}

Note. Items in bold are statistically significantly different from zero at $p<.05$, two-tailed.

${ }^{\mathrm{a}}$ For $r_{\mathrm{IQ}}, n=67$. 
Table 4

Means and Standard Deviations for Total Score for Combined Child and Adult Samples

\begin{tabular}{lccc}
\hline & Age Group (years) & $n$ & Mean \\
\hline $5-9$ & 158 & 17.28 & 3.97 \\
$10-14$ & 149 & 19.48 & 3.15 \\
$15-19$ & 69 & 22.35 & 2.49 \\
$20-24$ & 34 & 23.03 & 2.54 \\
$25-29$ & 17 & 22.29 & 1.86 \\
$30+$ & 53 & 22.36 & 3.11 \\
\hline
\end{tabular}


Table 5

Means, Standard Deviations, and Intercorrelations for the four TOL-4 measures, Age, and Verbal IQ for Children (Upper Diagonal) and Adults (Lower Diagonal)

\begin{tabular}{|c|c|c|c|c|c|c|c|c|c|c|}
\hline & & & & & & & & \multicolumn{3}{|c|}{ Children } \\
\hline & & \multicolumn{3}{|c|}{ Intercorrelations } & \multirow[b]{2}{*}{4} & \multirow[b]{2}{*}{5} & \multirow[b]{2}{*}{6} & \multirow[t]{2}{*}{ Mean } & \multirow[t]{2}{*}{$S D$} & \multirow[t]{2}{*}{$n$} \\
\hline & & 1 & 2 & 3 & & & & & & \\
\hline \multicolumn{2}{|l|}{ 1.Total } & & -0.02 & 0.83 & 0.86 & 0.42 & 0.11 & 18.70 & 3.82 & 334 \\
\hline \multicolumn{2}{|c|}{ 2. Planning Time } & 0.21 & & -0.01 & -0.04 & -0.35 & 0.004 & 9.20 & 6.33 & 334 \\
\hline 3. FST & & 0.68 & 0.26 & & 0.62 & 0.40 & 0.07 & 5.30 & 1.41 & 334 \\
\hline 4 Third & & 0.72 & 0.23 & 0.38 & & 0.37 & 0.09 & 6.90 & 1.38 & 334 \\
\hline 5. Age & & -0.07 & 0.18 & -0.10 & 0.03 & & -0.03 & 10.00 & 3.04 & 334 \\
\hline 6. IQ & & 0.002 & -0.03 & 0.09 & -0.14 & 0.09 & & 9.40 & 2.93 & 334 \\
\hline \multirow[t]{3}{*}{ Adults } & Mean & 22.50 & 8.10 & 6.70 & 8.30 & 27.70 & 12.50 & & & \\
\hline & $S D$ & 2.66 & 6.45 & 1.11 & 0.88 & 9.83 & 1.93 & & & \\
\hline & $n$ & 146 & 146 & 113 & 113 & 146 & 67 & & & \\
\hline
\end{tabular}

Note. Items in bold are statistically significantly different from zero at $p<.05$, two-tailed. 
Table 6

Estimates of Reliability for the Children and Adult Samples, for Items Scored on a 3-point Scale or Dichotomously

\begin{tabular}{|c|c|c|c|c|c|}
\hline \multirow[b]{2}{*}{ Sample } & \multicolumn{5}{|c|}{ Reliability Estimates } \\
\hline & Alpha & $95 \% \mathrm{CI}$ & Omega & glb & Lambda4 \\
\hline \multicolumn{6}{|l|}{ 3-point Scoring } \\
\hline Children & 0.58 & {$[.51, .65]$} & 0.64 & 0.67 & 0.65 \\
\hline Adults & 0.27 & {$[.08, .44]$} & 0.35 & 0.65 & 0.56 \\
\hline \multicolumn{6}{|c|}{ Dichotomous Scoring } \\
\hline Children & 0.43 & {$[.34, .52]$} & 0.48 & 0.52 & 0.50 \\
\hline Adults & 0.20 & {$[.01, .38]$} & 0.33 & 0.55 & 0.47 \\
\hline
\end{tabular}

Note. $\mathrm{CI}$ = confidence interval; glb = greatest lower bound. 

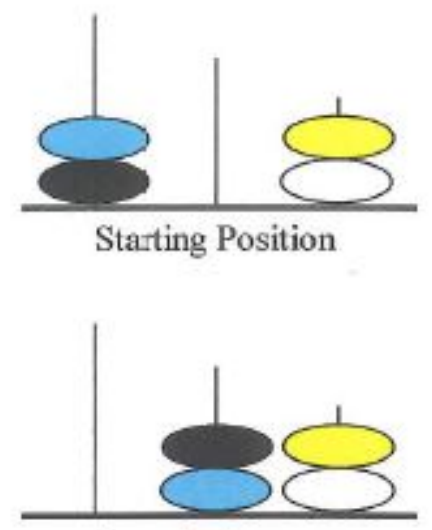

Item 1 - 2 moves

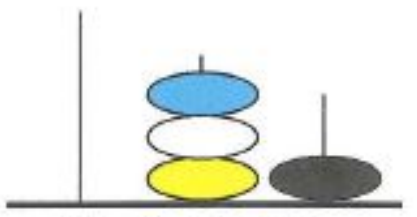

Item 3 - 4 moves

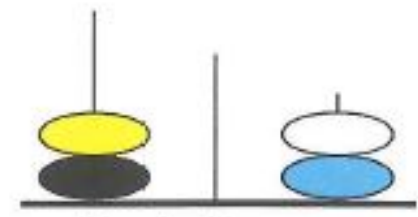

Item 5 - 5 moves

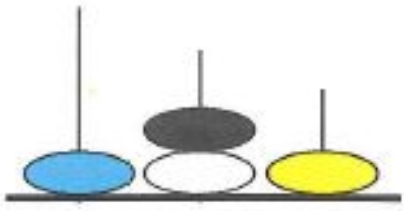

Item 7 - 6 moves

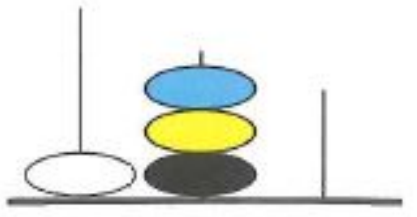

Item 9 - 9 moves

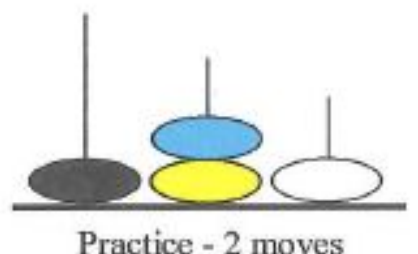

Practice - 2 moves

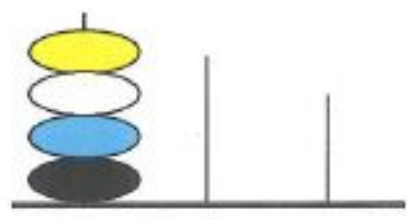

Item 2 - 3 moves
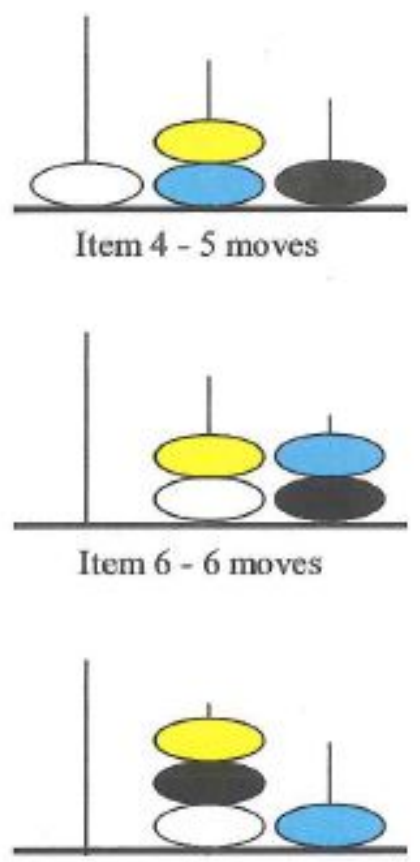

Item 8 - 7 moves

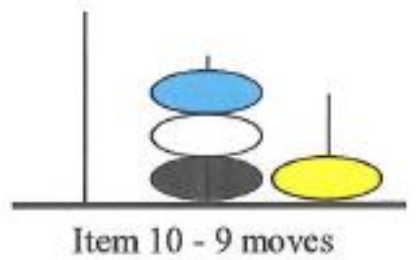

Supplementary Figure 1. Start and goal positions for the 10 items selected for the TOL4. 


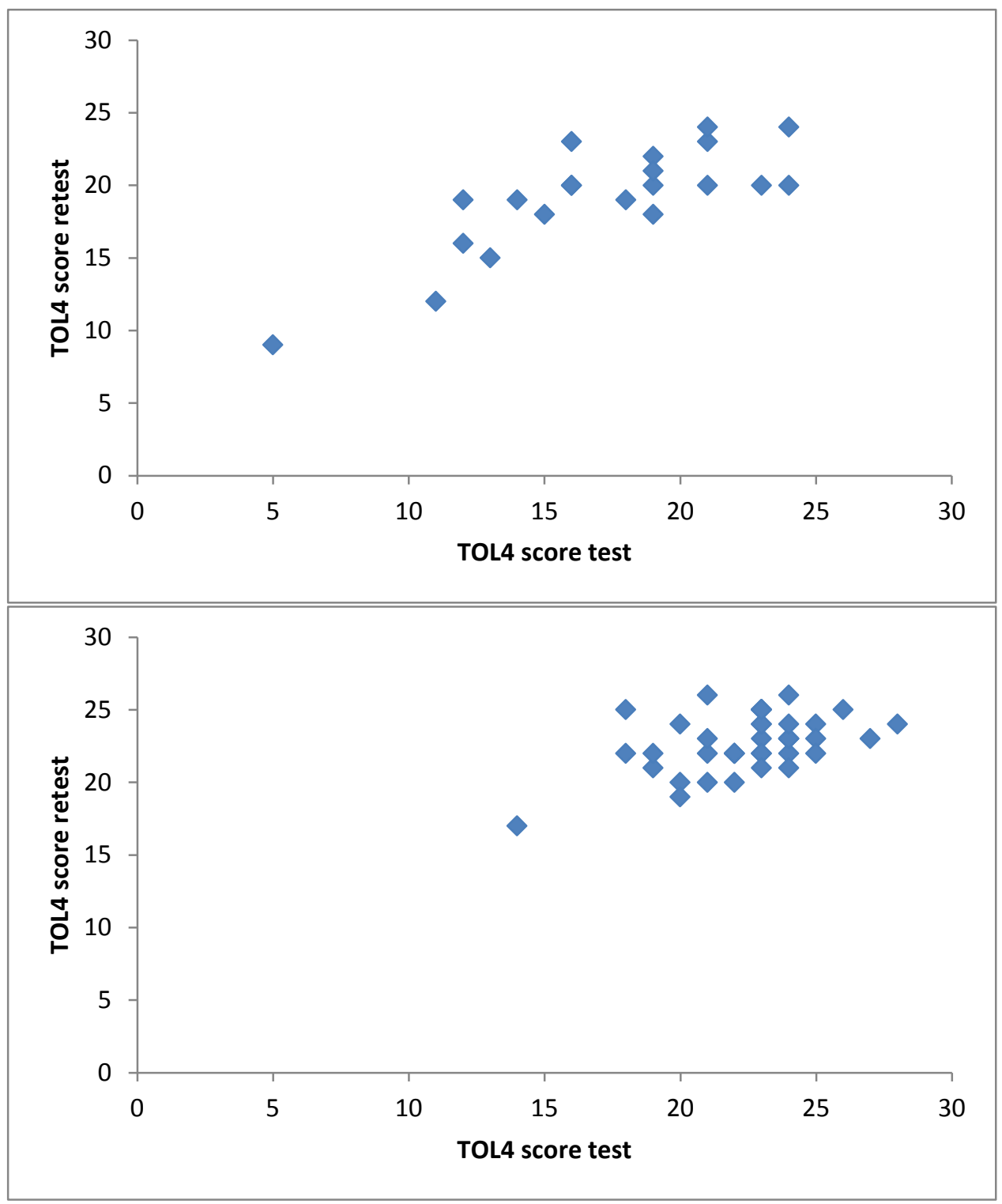

Supplementary Figure 2. Scatter plots of test-retest scores on the TOL4 for the children's sample (top panel) and the adult sample (bottom panel). 
Supplementary Table 1

Difficulty Levels and Correlations with Total Score of all Items and with Age for Items in Original Set

\begin{tabular}{|c|c|c|c|c|}
\hline Item & Description & Mean $(S D)$ & $r$ Total & rAge \\
\hline sc2a* & $\mathrm{F}$ & $2.91(0.29)$ & 0.55 & 0.32 \\
\hline $\mathrm{sc} 2 \mathrm{~b}$ & T3 & $2.91(0.29)$ & 0.55 & 0.52 \\
\hline sc2c & Т3 & $2.90(0.40)$ & 0.57 & 0.18 \\
\hline sc2d & $\mathrm{F}$ & $2.94(0.25)$ & 0.41 & 0.31 \\
\hline sc3a & $\mathrm{F}$ & $2.44(0.96)$ & 0.57 & 0.46 \\
\hline sc3b* & $\mathrm{T} 4$ & $2.79(0.64)$ & 0.78 & 0.25 \\
\hline sc3c & $\mathrm{F}$ & $2.90(0.30)$ & 0.31 & 0.13 \\
\hline sc3d & $\mathrm{F}$ & $2.90(0.30)$ & 0.22 & 0.13 \\
\hline sc4a & $\mathrm{F}$ & $2.50(0.83)$ & 0.71 & 0.25 \\
\hline sc $4 b^{*}$ & T3 & $2.56(0.93)$ & 0.65 & 0.44 \\
\hline $\mathrm{sc} 4 \mathrm{c}$ & T3 & $2.03(0.25)$ & 0.11 & -0.17 \\
\hline sc4d & $\mathrm{T} 4$ & 2.55 (0.89) & 0.64 & 0.23 \\
\hline sc $5 a^{*}$ & $\mathrm{~F}$ & $2.32(1.07)$ & 0.66 & 0.31 \\
\hline sc5b & Т3 & $2.35(1.04)$ & 0.67 & 0.34 \\
\hline $\operatorname{sc} 5 c^{*}$ & $\mathrm{~F}$ & $2.03(1.14)$ & 0.62 & 0.45 \\
\hline $\operatorname{sc} 5 d$ & $\mathrm{~F}$ & $2.29(1.07)$ & 0.43 & 0.03 \\
\hline sc6a & Т3 & $2.16(0.96)$ & 0.15 & -0.01 \\
\hline sc6b & $\mathrm{F}$ & $1.32(1.27)$ & 0.30 & 0.00 \\
\hline sc6c* & $\mathrm{F}$ & $2.00(1.20)$ & 0.63 & -0.10 \\
\hline sc6d* & $\mathrm{F}$ & $2.50(0.91)$ & 0.45 & -0.14 \\
\hline sc7a & T3 & 0.62 (1.09) & 0.27 & -0.09 \\
\hline sc7b* & $\mathrm{F}$ & $1.22(1.23)$ & 0.45 & 0.12 \\
\hline sc7c & T3 & $1.56(1.25)$ & 0.58 & -0.05 \\
\hline sc7d & $\mathrm{T} 4$ & $1.83(1.21)$ & 0.59 & -0.20 \\
\hline sc8a & $\mathrm{T} 4$ & $2.54(0.70)$ & 0.25 & 0.31 \\
\hline sc8b & T3 & $1.80(1.23)$ & 0.54 & 0.09 \\
\hline sc8c & $\mathrm{F}$ & $1.54(1.17)$ & 0.41 & -0.20 \\
\hline sc8d & $\mathrm{T} 4$ & 2.09 (1.15) & 0.63 & -0.26 \\
\hline sc9a* & T3 & $0.85(0.97)$ & 0.70 & -0.21 \\
\hline sc9b & $\mathrm{F}$ & 1.88 (1.17) & 0.22 & -0.16 \\
\hline sc9c* & Т3 & $0.17(0.51)$ & 0.49 & -0.24 \\
\hline sc9d & $\mathrm{F}$ & $0.71(0.98)$ & 0.23 & 0.12 \\
\hline sc10 & $\mathrm{F}$ & $0.78(1.08)$ & 0.53 & -0.24 \\
\hline
\end{tabular}


Note. $\mathrm{F}=$ flat ending goal position; $\mathrm{T}=$ tower ending goal position with the number indicating the number of discs. Coefficients in bold are statistically significant at $p<.05$ two-tailed. Items marked with asterisk are items selected for final version. 